\title{
Aplicação do Therapeutic Interventions Scoring System (TISS-28) em pós-operatório
}

\section{de cirurgia cardíaca}

\author{
Application of the Therapeutic Interventions Scoring System (TISS-28) in postoperative cardiac \\ surgery \\ Aplicación del Therapeutic Interventions Scoring System (TISS-28) en el postoperatorio de cirugía \\ cardíaca
}

\author{
Mariana Veiga da Silva \\ ORCID: https://orcid.org/0000-0001-9194-4266 \\ Universidade Veiga de Almeida, Brasil \\ E-mail: mvsmariana@hotmail.com.br \\ Nickson Scarpine Malheiros \\ ORCID: https://orcid.org/0000-0002-8092-1031 \\ Universidade Veiga de Almeida, Brasil \\ Universidade Federal do Rio de Janeiro, Brasil \\ E-mail: nicksonscarpini@gmail.com \\ Carlos Eduardo Peres Sampaio \\ ORCID: https://orcid.org/0000-0002-6770-7364 \\ Universidade Veiga de Almeida, Brasil \\ Universidade do Estado do Rio de Janeiro, Brasil \\ E-mail: carlosedusampa@yahoo.com \\ Luciana da Costa Nogueira Cerqueira \\ ORCID: https://orcid.org/0000-0003-1339-6828 \\ Universidade Veiga de Almeida, Brasil \\ Universidade Federal do Estado do Rio de Janeiro, Brasil \\ E-mail: luciana.nogueira7@hotmail.com \\ Anna Carolina das Neves Timoteo \\ ORCID: https://orcid.org/0000-0003-0136-1628 \\ Universidade Veiga de Almeida, Brasil \\ E-mail: Carolgica@gmail.com \\ Leonardo dos Santos Pereira \\ ORCID: https://orcid.org/0000-0002-8679-474X \\ Universidade Veiga de Almeida, Brasil \\ E-mail: leonardosp07@gmail.com
}

\begin{abstract}
Resumo
Objetivo: mensurar a carga horária de enfermagem ao paciente pós-operatório de cirurgia cardíaca, segundo o escore TISS-28. Questão norteadora: Qual a carga horaria de enfermagem ao paciente pós-operatório de cirurgia cardíaca? Metodologia: Trata-se de um estudo analítico de abordagem quantitativa, descritivo e exploratório. A pesquisa foi realizada em uma Unidade de Tratamento Intensivo adulto, privada, do Município de Cabo Frio no estado do Rio de Janeiro. Tendo como sujeitos da pesquisa os pacientes pós-operatórios de Cirurgia Cardíaca imediata. Resultados: Foram avaliados 87 pacientes internados em uma unidade de terapia intensiva. Em cada paciente, após a cirurgia, foi aplicado o escore TISS-28 para calcular o número de pontos obtidos a partir das intervenções necessárias em sua assistência. Conclusão: Foi obtido um elevado número da carga horaria de assistência de enfermagem, foi possível acompanhar a gravidade dos pacientes submetidos a cirurgias cardíacas.

Palavras-chave: Cirurgia cardíaca; Índice de gravidade de doença; Equipe de enfermagem; Carga de trabalho; Unidades de terapia intensiva.

Abstract

Objective: to measure the nursing workload for postoperative cardiac surgery patients, according to the TISS-28 score. Guiding question: What is the nursing workload for the postoperative cardiac surgery patient? Methodology: This is an analytical study with a quantitative, descriptive and exploratory approach. The research was carried out in a private adult intensive care unit in the city of Cabo Frio in the state of Rio de Janeiro. Having as research subjects the postoperative patients of immediate Cardiac Surgery. Results: 87 patients admitted to an intensive care unit were evaluated. In each patient, after the surgery, the TISS-28 score was applied to calculate the number of points obtained from the interventions necessary in their care. Conclusion: A high number of nursing care hours was obtained; it was possible to monitor the severity of patients undergoing cardiac surgery.
\end{abstract}


Keywords: Cardiac surgery; Severity of illness index; Nursing staff; Workload; Intensive care units.

\section{Resumen}

Objetivo: medir la carga de trabajo de enfermería para pacientes posoperatorios de cirugía cardíaca, según el puntaje TISS-28. Pregunta orientadora: ¿Cuál es la carga de trabajo de enfermería para el paciente posoperatorio de cirugía cardíaca? Metodología: Se trata de un estudio analítico con enfoque cuantitativo, descriptivo y exploratorio. La investigación se llevó a cabo en una unidad privada de cuidados intensivos para adultos en la ciudad de Cabo Frio en el estado de Río de Janeiro. Teniendo como sujetos de investigación a los pacientes postoperatorios de Cirugía Cardíaca inmediata. Resultados: se evaluaron 87 pacientes ingresados en una unidad de cuidados intensivos. En cada paciente, después de la cirugía, se aplicó el puntaje TISS-28 para calcular el número de puntos obtenidos de las intervenciones necesarias en su atención. Conclusión: Se obtuvo un alto número de horas de atención de enfermería, fue posible monitorear la gravedad de los pacientes sometidos a cirugía cardíaca.

Palabras clave: Cirugía cardíaca; Índice de gravedad de la enfermedad; Personal de enfermería; Carga de trabajo; Unidades de cuidados intensivos.

\section{Introdução}

Segundo a organização mundial de saúde (OMS), a síndrome coronariana aguda é a principal causa de mortes em todo o mundo e, consequentemente, no Brasil, atingindo um percentual em torno de $33 \%$ da mortalidade no país, sendo intensificada a busca por estratégias para o diagnóstico precoce e, por conseguinte, o início do tratamento adequado em tempo hábil. (Ribeiro, Goncalves, Borges, Loreto \& Amaral, 2019), (Somonucu, Demir, Karakut, Karakut \& Karabag, 2019)

Os sinais e sintomas mais comuns que caracterizam o surgimento de uma doença do coração são: cansaço, dor torácica, formigamento em membros superiores e dispneia. A queixa de queimação na região torácica também é comum. Entretanto, as síndromes coronarianas agudas podem ser assintomáticas, sendo sua primeira manifestação a morte súbita ou o infarto agudo do miocárdio. Neste sentido, medidas que visam prevenir essas doenças são primordiais para manter a qualidade e a perspectiva de vida do cliente, mesmo com os avanços tecnológicos na área cardiovascular. (De Castro, Santos, Goulart, Pereira, Staniak, Bittencourt, Lotufo \& Bensenor, 2019)

Nos últimos anos tem-se crescido muito o número de cirurgias cardíacas no Brasil, em especial as cirurgias de revascularização do miocárdio e correção de valvulopatias, entretanto, o número de óbitos tem crescido na mesma proporção. Pelo alto número de mortes, este é um procedimento arriscado, que compromete a vida do paciente, que deve ser considerado um paciente grave e passível de óbito, necessitando assim de uma assistência de enfermagem adequada, visando sua sobrevida. (Dessote, Figueiredo, Rodrigues, Furuya, Rossi \& Dantas, 2016)

A Unidade de terapia intensiva é o lugar mais apropriado para o paciente que foi submetido a algum tipo de cirurgia cardíaca, por ser o setor hospitalar que dispõe de tecnologia e equipamentos avançados, possuindo uma assistência mais especializada, onde contém meios para manter a vida e reestabelecer a saúde. (Freitas, Torres, Da Silva, Monteiro \& Vasconcelos, 2019)

Para que se mantenha um melhor acompanhamento do paciente pós-operado de cirurgia cardíaca e uma melhor avaliação do quadro clinico do paciente na unidade de terapia intensiva, o profissional enfermeiro deve ter um alto conhecimento técnico e científico que te exigirá rápidas tomadas de decisão. Em virtude das condições clinicas de cada paciente, a carga horaria de assistência de enfermagem no setor de terapia intensiva torna-se muito elevada. (Araujo, Henriques, Velloso, Queiroz \& Nonato, 2016)

A complexidade dos cuidados prestados está relacionada à gravidade do paciente, e isso faz necessitar um grande número de profissionais e uma alta carga horária de trabalho para um atendimento de qualidade. O Therapeutic Intervention Scoring System-28 (TISS-28) indica a necessidade de mais profissionais e uma estimativa da carga horária de enfermagem a partir da determinação da gravidade, da condição do paciente e do número de procedimentos a que ele está submetido. (Velozo, Garcia, Piva, Holmer, Cabral, Einloft, Bruno, Tonial, Costa \& Cabarro, 2017) 
O TISS-28 é um instrumento conhecido no mundo todo e é utilizado para mensurar a carga horária de assistência de enfermagem em pacientes críticos. São mensurados procedimentos realizados no paciente. Sendo assim o instrumento mede a intensidade do tratamento quantificando as intervenções médicas, mensurando assim, a carga horária de enfermagem, sendo o valor médio do escore para um enfermeiro é de 46 pontos em um turno de 08 horas, tendo cada ponto TISS-28 equivalente a 10,6 minutos de assistência. (Araujo et al, 2016), (Segura, Blanco, Vega \& Verzonis, 2015)

Diante do exposto, surgiram as seguintes questões norteadoras: Qual a carga horaria de enfermagem ao paciente pósoperatório de cirurgia cardíaca?

Foram delimitados como objetivo mensurar a carga horária de enfermagem ao paciente pós-operatório de cirurgia cardíaca, segundo o escore TISS-28.

Esta pesquisa justifica-se pela necessidade de melhorar a assistência prestada ao paciente no pós-operatório de cirurgia cardíaca, visto ser uma intervenção de grande complexidade e de alto índice de mortalidade, visando reduzir as complicações no pós operatório e reduzir a mortalidade, além de aprimorar a relação custo benefício na assistência à saúde.

\section{Metodologia}

Trata-se de um estudo analítico de abordagem quantitativa, descritivo e exploratório, visando identificar a gravidade do paciente de pós operatório de cirurgia cardíaca e a carga horária de enfermagem. A pesquisa quantitativa favorece a mensuração por meio de uma amostra que o represente estatisticamente. As características principais são: obedece a um plano pré-estabelecido, utiliza a teoria para desenvolver as hipóteses e as variáveis; verifica as relações entre as variáveis por métodos experimentais ou semi-experimentais. (Goncalves, Sampaio \& Junior, 2016), (Pereira, D.M Shitsuka, Parreira \& R Shitsuka, 2018)

Estudos descritivos procuram registrar, analisar, interpretar e descrever os fatos observados sem interferência do pesquisador. Enquanto o caráter exploratório visa maior proximidade do examinador, sem sua interferência, objetivando familiarizar-se com o fenômeno a ser investigado para maior exatidão dos resultados a serem alcançados. (Pradanov \& Freitas, 2013)

A pesquisa foi realizada em uma Unidade de Tratamento Intensivo (UTI) adulto, privada, do Município de Cabo Frio no estado do Rio de Janeiro, tendo como sujeitos da pesquisa os pacientes pós-operatórios de Cirurgia Cardíaca imediata. O cenário da pesquisa constituiu de uma UTI adulto, composta por dez leitos, sendo três destinados a pós-operatório de cirurgia cardíaca semanais.

Foram analisados 87 pacientes pós-operatórios de cirurgia cardíaca imediata, no período de março a outubro de 2020. Os tipos de cirurgia neste período foram: revascularização do miocárdio com circulação extracorpórea, revascularização do miocárdio sem circulação extracorpórea, troca de válvula mitral e troca de válvula aórtica.

O método de inclusão foi pós-operatório de cirurgia cardíaca imediata. Foi utilizado como método de exclusão pósoperatório de cirurgia cardíaca associada a qualquer outro tipo de cirurgia de grande porte, que poderia comprometer a avaliação da gravidade do paciente.

Os itens delimitados para determinação do perfil do pós-operatório de cirurgia cardíaca foram os parâmetros: faixa etária, gênero, história pregressa e o tipo de cirurgia cardíaca, referentes à internação na UTI no pós-operatório, e também foi utilizado o escore TISS-28, para classificar a gravidade dos pacientes correlacionando com a carga horária de enfermagem. Esta pesquisa foi no método não participante, utilizando os parâmetros das definições operacionais do TISS-28 que consistem em: atividade básica, suporte ventilatório, cardiovascular, renal, neurológico, metabólico e intervenção especifica. Estes parâmetros foram classificados em 04 (quatro) classes, através do somatório dos pontos atribuídos às atividades de enfermagem, utilizando a proposta de Kullen. (Guedes, Martins \& Chianca, 2015), (Chianca, Guedes, Souza, Morais \& Ercole, 
2015), (Gouzou, Karanikola, Lemonidou, Papathanassoglou \& Giannakopoulou, 2015)

O TISS-28 classifica os pacientes conforme os cuidados intensivos, propostos por Kullem, da seguinte forma: Classe I, de 00 a 20 pontos - pacientes fisiologicamente estáveis, mas requerendo observação profilática; Classe II, de 21 a 35 pontos - pacientes estáveis fisiologicamente, mas requerendo cuidados intensivos de enfermagem e monitorização contínua; Classe III, de 35 a 60 pontos - pacientes graves e instáveis hemodinamicamente; e Classe IV, mais de 60 pontos - pacientes com indicação compulsória de internação em UTI com assistência médica e de enfermagem contínua e especializada. O escore varia de um mínimo de pontos de zero a um máximo de 76 pontos. (Gouzou et al, 2015)

O estudo respeitou as exigências formais contidas na Resolução $n^{\circ}$ 466/12, que diz que toda pesquisa envolvendo seres humanos deve ser submetida à apreciação de um Comitê de Ética em Pesquisa, sendo aprovado pelo comitê de ética em pesquisa da Plataforma Brasil, sob o número do parecer de aprovação CAAE: 10985413.300005291.

Os dados quantitativos sofreram tratamento estatístico descritivo, foram tabulados e apresentados em tabelas desenvolvidas a partir de instrumentos de software estatístico EXCEL 2013. Análise desses dados envolveu estatística descritiva simples onde foram levantados resultados referentes a quantitativo absoluto, média simples e desvio padrão representado em porcentagem.

\section{Resultados}

Foram avaliados 87 pacientes internados em uma unidade de terapia intensiva, que passaram por uma cirurgia cardíaca. Foi aplicado o escore TISS-28 nas primeiras 24 horas imediatamente após o término da cirurgia, para identificar a gravidade de acordo com a classificação de cuidados intensivos de Kullem e carga horaria de enfermagem ideal para o pós operatório imediato de cirurgia cardíaca.

$\mathrm{Na}$ amostra observou-se uma predominância do gênero masculino, com 61 (70 \%) dos integrantes sendo do gênero masculino contra 26 (30\%) do gênero feminino. Em relação a idade, houve um predomínio no que se refere a idade entre 56 e 65 anos, com 49,5\% entre homens e mulheres. As idades entre 45 e 55 anos correspondem a 16 (18,3\%) dos indivíduos da amostra e os que tinham mais de 65 anos representaram $28(32,2 \%)$.

Tabela 1 - Características da população em estudo.

\begin{tabular}{lccc}
\hline Faixa Etária (anos) & $\begin{array}{c}\text { Gênero } \\
\text { Masculino \% (n) }\end{array}$ & $\begin{array}{c}\text { Gênero } \\
\text { Feminino \% (n) }\end{array}$ & $\begin{array}{l}\text { Amostra } \\
\%(\mathrm{n})\end{array}$ \\
\hline $45-55$ & $12,6(11)$ & $06(05)$ & $18,3(16)$ \\
$56-65$ & $36,7(32)$ & $12,6(11)$ & $49,4(43)$ \\
$>66$ & $20,6(18)$ & $11,5(10)$ & $32,2(28)$ \\
\hline Total & $70(61)$ & $30(26)$ & $100(87)$ \\
\hline
\end{tabular}

Fonte: Unidade de Tratamento Intensivo (UTI) adulto, Município de Cabo Frio no estado do Rio de Janeiro (2020).

Com relação à história pregressa, tiveram como destaque os pacientes com hipertensão arterial sistêmica (HAS) 92\% e dislipidemia 77\%, comparados com as outras comorbidades encontradas como: Diabetes Mellitus, Infarto Agudo do Miocárdio e Angina estável. 
Tabela 2 - História pregressa da população em estudo.

\begin{tabular}{cll}
\hline Comorbidades & N & $\%$ \\
\hline Hipertensão Arterial & 80 & 92 \\
Sistêmica & & 77 \\
Dislipidemia & 67 & 43 \\
Diabetes Mellitus & 38 & 55 \\
Infarto Agudo do & 48 & 08 \\
Miocárdio Angina Instável & & 07 \\
\hline
\end{tabular}

Fonte: Unidade de Tratamento Intensivo (UTI) adulto, Município de Cabo Frio no estado do Rio de Janeiro (2020).

Os tipos de cirurgias cardíaca encontradas no estudo foram: cirurgia de revascularização do miocárdio com circulação extracorpórea, representando $36(41,3 \%)$ dos pacientes da amostra total; revascularização do miocárdio sem circulação extracorpórea, realizados em $39(44,8 \%)$ dos pacientes; troca de válvula aórtica, em 8 (9,2\%) pacientes; e troca de válvula mitral, em $4(4,7 \%)$ pacientes.

Em cada paciente, após a cirurgia, foi aplicado o escore TISS-28 para calcular o número de pontos obtidos a partir das intervenções necessárias em sua assistência. Obtendo o número de pontos TISS-28, foi multiplicado por 10,6 por ser o valor em minutos de assistência correspondente a um ponto, e dividido por 60 para obter o valor em horas de assistência, e assim obter a carga horaria de enfermagem.

Tabela 3 - Carga horária de enfermagem para cada cirurgia.

\begin{tabular}{llll}
\hline Procedimentos & $\%(\mathrm{n})$ & Pontos TISS-28 & Carga horária (horas) \\
\hline $\begin{array}{l}\text { Revascularização do } \\
\text { miocárdio com CEC }\end{array}$ & $41,3(36)$ & 46 & 8,1 \\
Revascularização do & $44,8(39)$ & 37 & 6,5 \\
miocárdio sem CEC & & & \\
Troca de válvula Aórtica & $9,2(8)$ & 43 & 7,5 \\
Troca de válvula Mitral & $4,7(4)$ & 40 & 7,0 \\
\hline * CEC: Circulação extracorpórea Fonte: Unidade de Tratamento Intensivo (UTI) adulto, Município de Cabo Frio no \\
estado do Rio de Janeiro (2020).
\end{tabular}

De acordo com os dados apresentados na tabela 3, a partir dos cálculos dos dados coletados foi encontrado a cirurgia de revascularização do miocárdio com circulação extracorpórea, a cirurgia que exigiu mais assistência de enfermagem, com 8,1 horas de assistência por paciente; seguida da cirurgia de troca de válvula Aórtica, com 7,5 horas de assistência por paciente; Troca de válvula Mitral, com 7,0 horas por paciente; e revascularização do miocárdio, com 6,5 horas de assistência de enfermagem por paciente.

De acordo com a classificação de cuidados intensivos do TISS-28, proposta por Kullem, todos os pacientes avaliados nesta pesquisa se enquadram na classe III- de 35 a 45 pontos (pacientes graves e instáveis hemodinamicamente).

\section{Discussão}

Esse estudo demonstrou a carga horária ideal de enfermagem aplicado ao paciente de pós operatório de cirurgia cardíaca imediato, utilizando a escore de gravidade TISS 28. Foram avaliados um total de 87 pacientes submetidos a cirurgia cardíaca. Neste período de 2017, de março a outubro, no município de Cabo Frio foram realizados 4 tipos de cirurgia cardíaca, tendo um destaque na cirurgia de revascularização do miocárdio sem CEC com 44,8\%, seguido de revascularização do 
miocárdio sem CEC com 41,3\%.

O número de pacientes que são submetidos a cirurgias cardíacas para a correção de um problema de saúde tem se intensificado progressivamente, aumentando assim, os riscos de mortalidade no pós-cirurgico. No pré operatório uma avaliação minuciosa do histórico do paciente deve ser realizada, na intenção de prever as futuras complicações, uma vez que é grande o número de intervenções capazes de gerar alteração no paciente. (Cordeiro, De lima, Matos, Oliveira, Guimaraes, Carvalho \& Melo, 2017), (Cruz \& Estrada, 2017).

A média de idade foi de 60 anos, sendo a maioria do gênero masculino, estes dados são semelhantes a outros estudos realizados com pacientes submetidos a cirurgia cardíaca, onde prevaleceram o gênero masculino e idades em torno de 50 a 60 anos. ${ }^{2}$ Em contrapartida, este estudo apresentou uma prevalência menor de pacientes com faixa etária superior a 60 anos em comparação a outro estudo ${ }^{17}$. Porém, encontrou concordância com este mesmo estudo no que diz respeito a queixa dos pacientes, onde, $80,4 \%$ dos pacientes queixaram-se de dor no pós-operatório imediato, sendo um requisito para maior atenção da equipe de enfermagem. (Dessote $\boldsymbol{e t} \boldsymbol{a l}, 2016$ )

Os pacientes cardiopatas apresentam fatores de risco comuns, assim como em outros estudos, que apontam a hipertensão arterial e diabetes mellitus como as principais causas no desenvolvimento da placa arteriosclerótica. ${ }^{3,17}$ Esta pesquisa observou uma predominância nas comorbidades cardíacas, bem como história pregressa de hipertensão arterial sistêmica, diabetes mellitus e dislipidemia. Após a análise dos prontuários, foi visto que grande parte dos pacientes estudados relatou no pré-operatório fadiga relacionada ao estresse do dia-a-dia, bem como em outro estudo realizado. (Dessote $\boldsymbol{e t} \boldsymbol{a l}$, 2016)

Todos os pacientes estudados, independentemente do tipo de cirurgia realizada, se enquadraram na classe III proposta por Kullem, que são caracterizados pelo elevado número de pontos, e classificados como pacientes graves hemodinamicamente, requerendo uma atenção e uma assistência de enfermagem especializada.

Sendo a opção de escolha em muitos casos de cardiopatia, a cirurgia cardíaca, apesar do avanço no tratamento de doenças cardiovasculares tem crescido muito nos últimos anos e pelo fato de a abordagem ser minimamente invasiva, é considerada uma cirurgia de grande porte, necessitando muitas vezes de circulação extracorpórea. (Dessote $\boldsymbol{e t ~ a l , ~ 2 0 1 6 ) ~}$

Assim observou-se neste estudo que a cirurgia de revascularização do miocárdio com circulação extracorpórea obteve a maior carga horaria de enfermagem ( 8,1 horas) entre as cirurgias estudadas, necessitando de muitas intervenções de enfermagem; seguido de troca de válvula aórtica (7,5 horas) e troca de válvula mitral (7,0 horas), tendo como o procedimento com menor carga horaria de enfermagem a revascularização sem CEC, com um total de 6,5 horas.

Uma carga horária de enfermagem que seja exaustiva para o profissional contribui para baixas taxas de conformidade, como a higienização das mãos e, consequentemente, cuidados abaixo do padrão de qualidade em relação ao controle de infecções, podendo comprometer muito mais o paciente pós-cirúrgico, onde já apresenta uma porta de entrada para estes micoorganismos. (Nieri, Manousaki, Kalafati, Padilha, Stafseth, Katsoulas, Matziou \& Giannakopoulou, 2018)

Um estudo analisado, realizado no ano de 2017 através de uma revisão integrativa acerca da assistência perioperatória de enfermagem a pacientes portadores de cardiopatia, afirma que no decorrer de todo o processo que o paciente passa para ser submetido a uma cirurgia cardíaca, o enfermeiro presta uma assistência ininterrompida e é fundamental, que tenha um número de profissionais adequado para atender a demanda do paciente para evitar elou reverter qualquer complicação apresentada no pós-cirúrgico. (W. L. A.V. Silva, Barros, Dos Santos, L. A. Silva \& Miranda, 2018)

Os scores obtidos através da aplicação da sistemática TISS-28 fornecem subsídios para que o gerente de enfermagem possa interpretar a sua realidade de trabalho, objetivando gerar uma boa assistência para seus clientes. A correlação entre a complexidade dos pacientes da UTI e a demanda de cuidados de enfermagem auxiliam na adequação do quantitativo da equipe de enfermagem. (Beccaria, Melara, Pereira, Calil \& Trevisan, 2010) 
Após o término da cirurgia, é imprescindível a presença do enfermeiro na admissão do paciente na unidade de pós operatória de cirurgia cardíaca, além do seu acompanhamento contínuo na realização de procedimentos de alta complexidade, pois o paciente pode desestabilizar rapidamente, requerendo cuidados intensivos imediatos. (Somuncu et al, 2019), (Lopes, Castro, Braga, Gomes, Silva, Brandao \& Gomes, 2019)

\section{Conclusão}

O TISS-28 conseguiu prever, como o esperado, as exigências que a gravidade dos pacientes exigiu em relação à carga horaria de assistência de enfermagem. O escore permitiu também classificar, de acordo com a proposta de Kullem, a classe à qual os pacientes se enquadraram. Embora se tenha obtido um número alto da carga horaria de assistência de enfermagem, o escore não apresenta pontuação para algumas atuações de enfermagem como procedimentos com a higiene, suporte e atenção aos familiares, além das tarefas gerenciais e administrativas. Contudo foi possível acompanhar a gravidade dos pacientes submetidos a cirurgias cardíacas, bem como a evolução da gravidade do paciente e as intervenções de enfermagem diante das intercorrências das primeiras 24 horas.

Logo, definiu-se que o procedimento no qual demandou uma maior carga horaria media a revascularização do miocárdio com CEC, com 8,1 horas e 46 pontos no escore TISS-28 e como procedimento que exigiu uma menor carga horaria media a revascularização do miocárdio sem CEC, com 6,5 horas e 37 pontos no escore TISS-28.

Os resultados deste estudo nos trouxeram informações primordiais para analisar e constatar a demanda dos cuidados de enfermagem e refletir sobre a carga horaria de assistência de enfermagem para os pacientes submetidos a cirurgias cardíacas.

Com o advento de novas tecnologias e recursos terapêuticos para facilitar o tratamento e recuperação do paciente em UTI, a enfermagem está utilizando cada vez mais escores que avaliam a gravidade dos pacientes, com o intuito de planejar uma assistência adequada às necessidades do cliente. Por meio desta pesquisa utilizando o TISS-28 em pós operatório de cirurgia cardíaca é possível identificar a gravidade e a carga horária de enfermagem, possibilitando para futuros trabalhos uma melhoria nas intervenções de enfermagem no pós operatório de cirurgia cardíaca, podendo assim contribuir para o planejamento da prática assistencial.

\section{Referências}

Araujo M. T., Henriques V. B., Velloso I. S. C., Queiroz C. F. \& Nonato L. F. (2016). Carga de trabalho e custo de uma equipe de enfermagem em terapia intensiva. Arq. Ciênc. Saúde. 23(4) 21-6. https://www.cienciasdasaude.famerp.br/index.php/racs/article/view/385/232

Barros S. R., Bandeira M. M. \& Leite J. C. R. A. P. (2019). Principais complicações da circulação extracorpórea em cirurgias cardíacas em um hospital da Região do Norte. Saber Científico. 8(1) 103-110. http://periodicos.saolucas.edu.br/index.php/resc/article/view/1267

Beccaria L. M., Melara S. V. G., Pereira R. A. M., Calil A. S. G. \& Trevisan M. A (2010). Horas de cuidados de enfermagem em UTI: utilização do sistema de pontuação de intervenções terapêuticas. Arq Ciênc Saúde. 17(1):48-53. http://repositorio-racs.famerp.br/racs_ol/vol-17-1/ID8L_jan-mar_2010.pdf

Chianca T. C. M., Guedes H. M., Souza K. M., Morais S. S. \& Ercole F. F. (2015). Avaliação da gracidade de pacientes internados em clinicas de um hospital. Cienc. Enferm. 21(3), 11-21. http://dx.doi.org/10.4067/S0717-95532015000300002.

Cruz I. L. \& Estrada J. C. C. (2017). Cuidados Al Adulto Postoperado En La Administración De Insulina. Enf Neurol. 16(2): 78-90. https://docs.bvsalud.org/biblioref/2020/02/966170/cuidados-al-adulto-posoperado.pdf

De Castro L. T., Santos I. S., Goulart A. C., Pereira A. C., Staniak H. L., Bittencourt M. S., Lotufo P. A. \& Bensenor I. M. (2019). A Troponina I de Alta Sensibilidade Elevada na Fase Estabilizada após Síndrome Coronariana Aguda Prevê Mortalidade por Todas as Causas e Mortalidade Cardiovascular em uma População Altamente Miscigenada: Uma Coorte de 7 Anos. Arq. Bras. Cardiol. 112(3):230-237. 10.5935/abc.20180268.

Dessote C. A. M, Figueiredo M. L, Rodrigues H. F. Furuya R. K., Rossi L. A. \& Dantas R. A. S. (2016). Classificação dos pacientes segundo o risco de complicações e mortalidade após cirurgias cardíacas eletivas. Rev. Eletr. Enferm. 18:e1140. http://dx.doi.org/10.5216/ree.v18.37736

Freitas K. O., Torres R. S. C., Da Silva S. E. D., Monteiro G. O. \& Vasconcelos E. V. (2019). Representações Sociais de Familiares de Pacientes em Unidades de Terapia Intensiva: Implicações no Cuidado de Si. Revista de Pesquisa: Cuidado é Fundamental Online. 11(3): 664-71. https://doi.org/10.9789/21755361.2019.v11i3.664-671 
Research, Society and Development, v. 10, n. 10, e490101019221, 2021

(CC BY 4.0) | ISSN 2525-3409 | DOI: http://dx.doi.org/10.33448/rsd-v10i10.19221

Gonçalves, R. A., Sampaio, C., \& Júnior, H. (2016). Determinação dos fatores da suspensão de cirurgia e suas contribuições para assistência de enfermagem. Revista De Pesquisa Cuidado é Fundamental Online, 8(3), 4813-4820. https://doi.org/10.9789/2175-5361.2016.v8i3.4813-4820

Gouzou M., Karanikola M., Lemonidou C., Papathanassoglou E. \& Giannakopoulou M. (2015). Margarita Avaliação da satisfação profissional e carga laboral das equipes de enfermagem nas Unidades de Cuidados Coronarianos Gregas. Rev Esc Enferm USP. 49(Esp):15-21. 10.1590/S0080-6234201500000003

Cordeiro A. L. L., De Lima A. S. S., Matos I. C. O., Oliveira L. V. B., Guimaraes A. R., Carvalho S. O. \& Melo T. A. (2017). Análise do tempo de ventilação mecânica e internamento em pacientes submetidos a cirurgia cardíaca. ABCS Health. 42(1):3-7. https://doi.org/10.7322/abcshs.v42i1.942

Guedes H. M., Martins J. C. A. \& Chianca T. C. M. (2015). Valor de predição do Sistema de Triagem de Manchester: avaliação dos desfechos clínicos de pacientes. Rev. Bras. Enferm. 68(1):45-51. https://doi.org/10.1590/0034-7167.2015680107p.

Lopes R. O. P., Castro J. N., Braga C. S. C., Gomes D. V., Silva J. R., Brandao R. C. \& Gomes M. A. (2019). Complicações do pós-operatório imediato de cirurgia cardíaca eletiva: estudo transverds. Revista de Enfermagem Referência. 4 (22): 22-37. https://doi.org/10.12707/RIV19042

Nieri, A. S., Manousaki, K., Kalafati, M., Padilha, K. G., Stafseth, S. K., Katsoulas, T., Matziou, V., \& Giannakopoulou, M. (2018). Validation of the nursing workload scoring systems "Nursing Activities Score" (NAS), and "Therapeutic Intervention Scoring System for Critically Ill Children" (TISS-C) in a Greek Paediatric Intensive Care Unit. Intensive \& critical care nursing, 48, 3-9. https://doi.org/10.1016/j.iccn.2018.03.005

Pereira A. S. et al. (2018). Metodologia da pesquisa científica. UFSM. 5.3)

Pradanov C. C. \& Freitas E. C. (2013). Metodologia do trabalho cientifico: Métodos e técnicas da pesquisa e do trabalho acadêmico. Feevale. http://www.feevale.br/Comum/midias/8807f05a-14d0-4d5b-b1ad-1538f3aef538/E-book\%20Metodologia\%20do\%20Trabalho\%20Cientifico.pdf

Ribeiro K. R. A, Gonçalves F. A. F, Borges M. M., Loreto R. G. O. \& Amaral M. S. (2019). Pós-Operatório de Revascularização do Miocárdio: Possíveis Diagnósticos e Intervenções de Enfermagem. Rev Fund Care Online. 11(3):801-808. http:// dx.doi.org/10.9789/2175-5361.2019.v11i3.801-808.

Segura S. M. R., Blanco J. P., Vega M. H. G. \& Verzonis N. M. (2015). Aplicación del sistema pronóstico TISS-28 en salas de atención al grave. Rev Cub Med Int Emerg. 14(1):53-66. https://www.medigraphic.com/pdfs/revcubmedinteme/cie-2015/cie151g.pdf

Silva W. L. A. V., Barros A. T. L., dos Santos, R. D., Silva, L. A., \& Miranda, L. N. (2018). Cirurgias cardíacas: assistência de enfermagem a portadores de cardiopatia no período perioperatório. Caderno De Graduação - Ciências Biológicas E Da Saúde - UNIT - ALAGOAS,4(2), 323. https://periodicos.set.edu.br/fitsbiosaude/article/view/4565

Somuncu M. U. D., Demir A. R., Karakurt S. T., Karakurt, H. \& Karabag T. (2019). Desfecho Cardiovascular em Longo Prazo com Base na Capacidade de Resposta à Aspirina e ao Clopidogrel em Pacientes Jovens com Infarto do Miocárdio com Elevação do Segmento ST. Arq. Bras. Cardiol. 112(2):138-146. $10.5935 / \mathrm{abc} .20180251$.

Velozo K. D. S., Garcia P. C. R., Piva, J. P., Holmer F. H., Cabral D. D., Einloft P. R., Bruno F., Tonial C. T., Costa C. A. D., \& Canabarro S. T. (2017). Escores TISS-28 versus NEMS para dimensionar a equipe de enfermagem em unidade de terapia intensiva pediátrica. Einstein. 15(4):470-5. https://www.scielo.br/j/eins/a/NCZkzGD3dQpNMmgqDH6GGNb/?format=pdf\&lang=pt 